\title{
The Role of the Limbal Cornea in Vision
}

\author{
DAVID MILLER, NEAL ATEBARA, ROBERT STEGMANN \\ Boston, USA and Medunsa, South Africa
}

\begin{abstract}
Summary
The eagle retina is bifoveal. The temporal fovea, which can resolve targets subtending half a minute or less must depend upon its peripheral cornea for such sharp resolution. Our studies showed the eagle cornea to be thinner, scatter less light and be more spherical than the human cornea. Further experimental studies determined the resolving power of the human peripheral cornea. Using, normal subjects, pinholes placed at the edge $8 \mathrm{~mm}$ dilated pupils yielded $20 / 30$ visual acuity and normal contrast sensitivity measurements. Thus it is recommended that optical iridectomy be considered, in appropriate cases of corneal blindness with patches of clear peripheral cornea when no donor corneal material is available.
\end{abstract}

A paper entitled the optics of the corneal periphery must sound strange. After all, everyone knows that man's vision, as well as that of most animals, depends upon the precision optics of the central cornea. ${ }^{1}$

But, are there animals that may also have excellent peripheral corneal optics?

The eagle is capable of a vision of $20 / 10$ or even $20 / 5 .^{2}$ Its retina is unusual in that it has two foveas. It is the temporal fovea, which receives focussed light through the peripheral nasal cornea, and is responsible for the high degree of resolution. Thus, we were interested in exploring the optics of the eagle cornea.

However, we also had a second objective i.e. to explore the peripheral corneal optics in man. The reason for such interest relates to the high incidence of corneal blindness in the third world. For example it is estimated that in the third world, six to nine million are blind from trachoma and another 100 million have serious visual impairment from the same disease.
Xerophthalmia produces corneal blindness in 250,500 children in Asia every year. ${ }^{3}$ Unhappily, the third world does not have a sufficient number of corneal surgeons or a sufficient amount of donor material to treat those blind from corneal disease. Perhaps 25 per cent of these blind have a patch of peripheral clear cornea. In such cases, an optical iridectomy could be useful. Therefore, we were prompted to find out the optical potential of the peripheral cornea.

\section{Method}

In order to determine the optical properties of the eagle cornea, the Zoological Garden in Pretoria, South Africa was requested to bring to the eye clinic of the Medical University of Southern Africa (Medunsa) an adult Batteleur eagle. The eagle was sedated, intubated and kept under general anesthesia while its corneae were examined. A photokeratoscope (Nidek, Japan) was then used to take placido disc photographs of the cornea (Fig. 1). Finally, an ophthalmometer (Zeiss, 


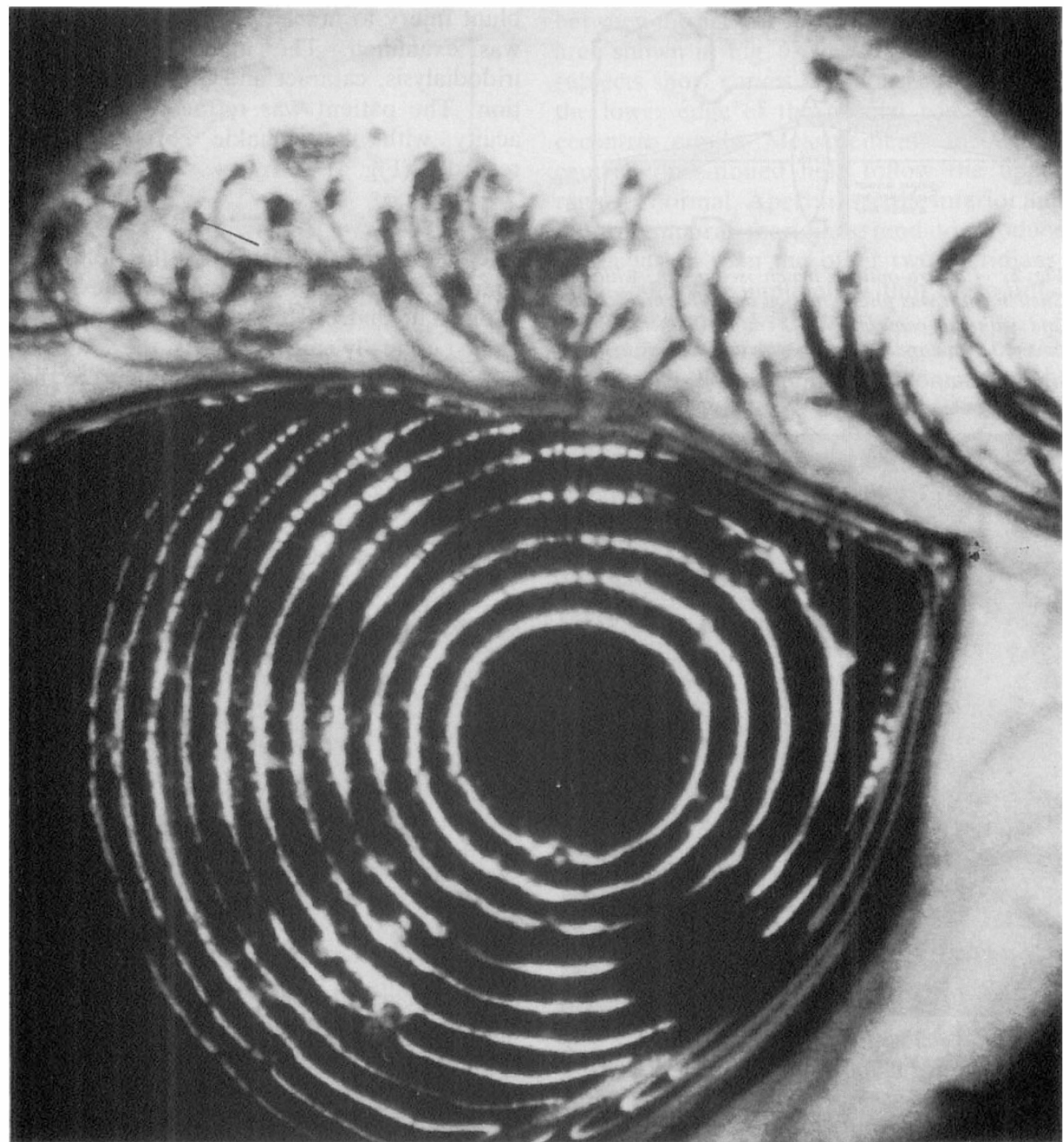

Fig. 1. Keratoscopic photo of cornea of Batteleur eagle under general anaesthesia. The irregularity of the central rings is due to a temporary drying episode.

Germany) was used to evaluate the central cornea of the eagle.

Figure 2 shows an optical experiment in which a camera was placed on an optical bench with an illuminated Snellen chart as the object. An opaque screen with a $3 \mathrm{~mm}$ aperture was positioned in front of the camera lens.

Pictures were taken of the image of the
Snellen chart, with the aperture in the screen placed over the centre of the camera lens and with the aperture eccentrically placed $(4 \mathrm{~mm}$ from the centre).

In another set of experiments, an opaque screen with a small aperture $(1.6 \mathrm{~mm})$ was used to simulate an optical iridectomy. The device was held close (about $2 \mathrm{~mm}$ ) to the cornea. The pupil was dilated to $8 \mathrm{~mm}$ and the 


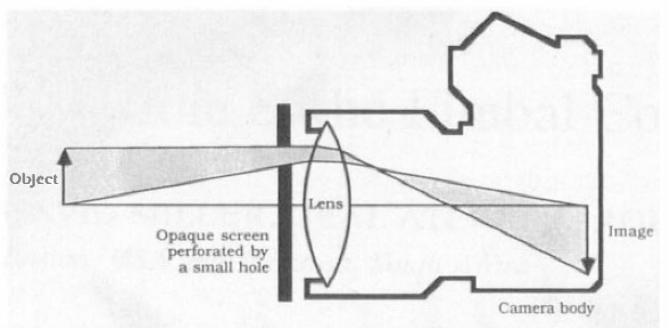

Fig. 2. Camera-optical bench setup, in which a Snellen chart was photographed in the normal fashion and with the camera lens $(2 \mathrm{~cm}$ in diameter) being covered by an opaque screen with an eccentric aperture (3 $\mathrm{mm})$.

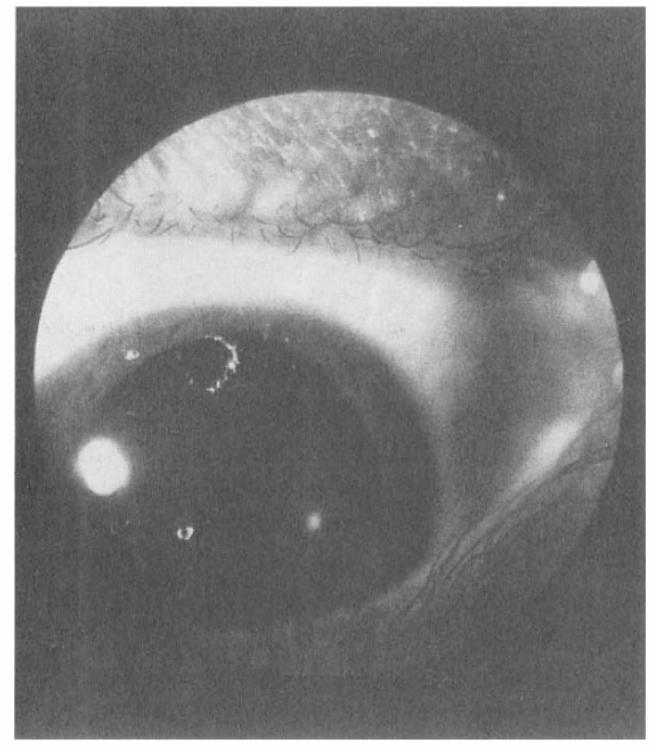

Fig. 3. Photograph superimposing the position of the eccentric hole on the $8 \mathrm{~mm}$ dilated pupil of the subject.

pinhole was carefully positioned over the peripheral cornea at the edge of the dilated pupil. The exact position of the pinhole with respect to the pupil was verified by photographically superimposing the position of the pinhole with the position of the subjects pupil (Fig. 3). The pinhole was positioned just inside the edge of the maximally dilated $(8 \mathrm{~mm})$ pupil, and at various meridians (3, 6 and 9 o'clock).

With the pinhole in these positions, as well as over the pupil centre, Snellen acuity and contrast sensitivity were recorded using the Vision Contrast Test System (Vistech Consultants, Inc, Dayton, Ohio) in two subjects.

Finally, a patient who suffered a severe blunt injury to her left eye from a bullwhip was examined. The injury produced an iridodialysis, cataract and mild lens dislocation. The patient was refracted and visual acuity with the aphakic correction was recorded (Fig. 4).

\section{Results}

The eagle cornea displayed the following characteristics. The corneal thickness was about half that of the human and appeared almost optically empty. That is, there was far less backscattering of light, compared to that in humans. The central corneal curvature was 36 diopters and spherical and the mires reflections were very regular, after the lids were used to lubricate the cornea. The keratoscopic photo (Fig. 1) showed the peripheral cornea to be spherical and regular. Unhappily, the

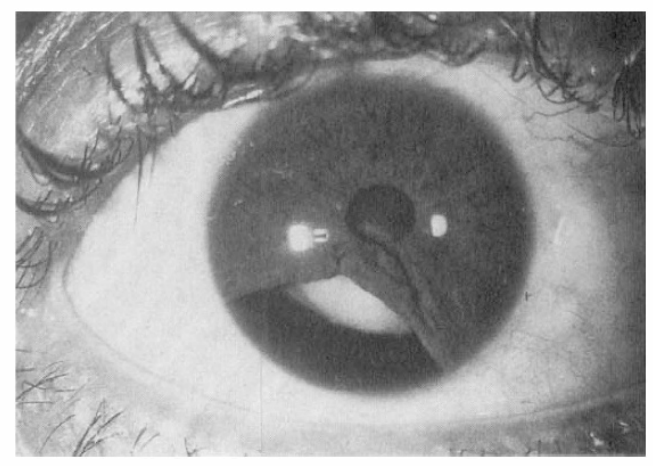

Fig. 4. Photograph of the eye of a patient with a bullwhip induced cataract, and iridodialysis (courtesy Robert Stegmann).

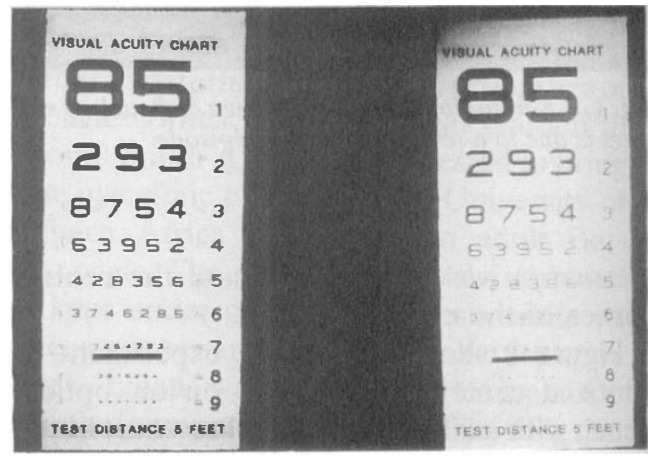

Fig. 5. The photograph of the Snellen chart at the top was taken in the normal manner. The photograph at the bottom was taken with the same camera, but through the eccentric aperture over the camera lens. 


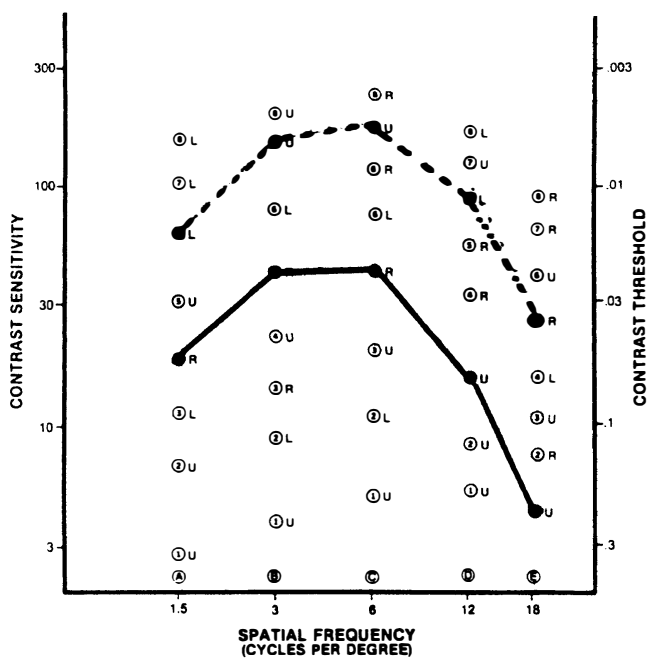

Fig. 6. Contrast sensitivity recording of one of the subjects viewing the target through (a) a $1.6 \mathrm{~mm}$ eccentric hole and (b) a $1.6 \mathrm{~mm}$ hole centrally placed over the dilated pupil.

central rings look irregular in the photo, because of a temporary drying episode. The pupil remained at a diameter of $6 \mathrm{~mm}$ during the examination and did not respond to light. The curvature of crystalline lens was flatter than in man.

Figure 5, (left), is the control photo of the Snellen chart, taken with the small aperture of the opaque screen positioned over the centre of the camera lens. Figure 5, (right), is the picture of the chart taken through the same aperature, this time positioned over the periphery of the camera lens. Note, most lines of the chart can be seen, although contrast using the peripheral aperture is poorer. Visual acuity for each of the two subjects looking through the opaque screen with the eccentric aperture (simulated peripheral optical iridectomy) was $20 / 30$. This was true whether the hole was placed at 3,6 or 9 o'clock. Visual acuity, with the same small aperture placed over the pupil centre was $20 / 20$ for each subject.

Contrast sensitivity was plotted against spatial frequency (in cycles per degree) to produce a contrast sensitivity curve. The contrast sensitivity of $90 \%$ of the normal population between the ages of 10 and 70 falls in the gray area shown in Fig. 6. The results from both subjects show curves that consistently fall at the lower edge of the normal zone for the eccentric pupils. Measurements through a centrally positioned hole follow the upper range of normal. Apertures at the inferior and infero-temporal meridians tend to produce better vision than the other two meridians, falling almost completely within the normal range for contrast sensitivity.

The best corrected visual acuity of the patient with the traumatic iridodialysis and cataract was $20 / 60$.

\section{Discussion}

It would appear that the extraordinary vision of the temporal fovea of the eagle is due to

(a) decreased light scattering by the cornea as compared to man

(b) a spherical corneal periphery

(c) an enlarged retina image due to a significantly longer eye.

The experiments on human subjects further tell us that in the healthy eye a small optical iridectomy placed over the corneal periphery yield a Snellen acuity of 20/30 and normal contrast sensitivity.

One could legitimately ask if the good visual function is due to excellent peripheral corneal optics or if it is produced by the pinhole effect. Since the pinhole can only correct a finite amount of blurring, ${ }^{4}$ one would assume that both peripheral corneal optics and the pinhole contribute to the effect.

We recommend that optical iridectomy be considered in dealing with cases of corneal blindness, if a clear patch of peripheral cornea is present and if corneal surgeons and corneal donor material are not available.

\section{References}

${ }^{1}$ Miller D and Carter J: A proposed new division of corneal function in the Cornea, Transactions of the World Congress on the Cornea III. Ed Cavanagh HD, Raven Press, NY 1987, p. 155.

2 Schlaer R: An Eagle's Eye: Quality of the Retinal Image. Science 1972, 176: 922.

${ }^{3}$ World Blindness and Its Preventions, Oxford University, Press, Oxford England, 1984, pp. 4-12.

${ }^{4}$ Miller D and Johnson R: Quantificaiton of the Pinhole Effect. Surv Ophthalmol 1977, 21: 147. 